\title{
9
}

José López-Tafall Bascuñana*

\section{ALGUNAS CLAVES SOBRE LAS LEYES DE TRANSICIÓN ENERGÉTICA Y SU POSIBLE APLICACIÓN EN ESPAÑA}

La necesidad de una norma de rango legal que impulse y gobierne la transición energética es un aspecto largamente contemplado en España pero que aún no se ha desarrollado. La experiencia reciente de otros países europeos, o el anuncio de una futura ley europea de cambio climático, sin duda, impulsará el debate en nuestro país. El objetivo de este artículo es presentar una visión general de esta temática, con el objeto de ayudar al lector profano a tener un conocimiento básico de la problemática. El método empleado es analizar las razones para una ley y su posible contenido, así como aportar información sobre puntos conflictivos que, sin duda, serán objeto de discusión. La conclusión principal es que es necesaria una ley en España que cuente con un consenso similar al que impulsó a otros procesos de cambio estructural en nuestro país.

Palabras clave: descarbonización, cambio climático, energía, renovables, emisiones.

Clasificación JEL: Q40, Q52, Q54.

\section{Introducción}

El pasado 11 de diciembre, tan solo diez días después de ser votada por el Parlamento Europeo, la nueva Comisión Europea presentó su Comunicación sobre el Pacto Verde Europeo ${ }^{1}$, que describe la hoja de ruta que se pretende impulsar para alcanzar la descarbonización de la economía europea. Dentro de la misma aparecía una primera medida: aprobar antes de 100 días una propuesta de «Ley europea del clima».

\footnotetext{
* Técnico Comercial y Economista del Estado. Versión de enero de 2020.

DOI: https://doi.org/10.32796/bice.2020.3120.6964

Comisión Europea, 11/12/2019. Comunicación de la Comisión Europea sobre el Pacto Verde Europeo, COM (2019) 640 final.
}

Si bien esta propuesta es novedosa a nivel comunitario, no lo es a nivel nacional. Desde que Reino Unido aprobara en 2008, bajo liderazgo conservador, su Climate Change $\mathrm{Act}^{2}$, numerosos países han aprobado normas con rango de ley para impulsar aspectos relacionados con el cambio climático ${ }^{3}$. Muchos de ellos son socios comunitarios o países con importantes relaciones con España: este es el caso de Francia, Suecia o México, entre otros.

El reciente protagonismo español en la Conferencia de las Partes (COP 25), celebrada en Madrid, ha vuelto a poner de manifiesto la paradoja que supone que España carezca de $\triangleright$

2 http://www.legislation.gov.uk/ukpga/2008/27/contents

3 En septiembre de 2018 existían en el mundo más de 1.500 leyes y normas de rango ejecutivo vinculadas con el cambio climático, de las que el $42 \%$ eran leyes (Averchenkova, 2019). 
una ley similar. Tras anuncios, anteproyectos y declaraciones casi unánimes de los partidos políticos sobre la necesidad de tal ley, cabe esperar que, por fin, su debate y aprobación sea inminente.

El objetivo de este artículo es ilustrar de modo muy resumido aspectos básicos sobre esa figura, quizá más mencionada que conocida. Tratará aspectos relevantes sobre por qué es necesaria una ley y qué contenidos mínimos debería incluir. Siendo, como es aún hoy, un tema abierto a discusión, concluirá repasando algunos de los puntos conflictivos que se han utilizado tradicionalmente en los debates en nuestro país, intentando aportar datos para que el lector realice su propio análisis crítico.

\section{Objetivo y necesidad de una ley}

La primera cuestión que un país debe decidir es para qué necesita una ley de cambio climático. Tal pregunta equivale a cuestionarse qué objetivos se desean alcanzar y, por tanto, qué nivel de ambición deben fijarse como sociedad. La experiencia internacional es muy variada, por lo que es aconsejable centrarse en los países de nuestro entorno.

La ley pionera relevante fue la ley británica de 2008 , con un objetivo muy enfocado en las emisiones de $\mathrm{CO}_{2}$. Se fijaban objetivos de reducción de emisiones a 2050, pero también objetivos quinquenales (carbon budget), plazo elegido para evitar quedar condicionado por el ciclo político. Buena parte del contenido de la ley consistía en desarrollar los instrumentos para hacer posible esa reducción, tanto desde su monitorización (por un organismo independiente) como desde su ejecución (mediante el fomento de esquemas de permisos negociables).
Con el paso del tiempo y, sobre todo, tras el Acuerdo de París, las leyes europeas de cambio climático han mudado hacia objetivos más amplios y estructurales. Un ejemplo reciente es la ley francesa de 2015 de «Transición Energética para el Crecimiento Verde " ${ }^{4}$, que inspira el anteproyecto de ley española sometido a consulta en enero de 2019. Atendiendo a su exposición de motivos, llama la atención que casi ni menciona los gases de efecto invernadero o el calentamiento climático. Su objeto es ya mucho más amplio: «comprometer a todo el país en el camino de un crecimiento verde creador de riqueza, empleos sostenibles y de progreso».

La ley trasciende así del ámbito climático para convertirse en un objetivo nacional y transversal, en un programa de cambio estructural de toda la economía francesa. $Y$ se hace para impulsar el «crecimiento», combatir el paro o reducir la factura energética, entre otros. ¿Cuál es la herramienta para ello? Crear un nuevo modelo energético, la «transición energética».

El único texto conocido del anteproyecto español integra ambas posiciones y encaja perfectamente con las tendencias europeas. Su objetivo es doble: por un lado, fijar la reducción de emisiones de gases de efecto invernadero (en adelante, GEI) a 2030 y 2050 , pero, por otro lado, impulsando un programa de transición energética que afecte al conjunto de la economía y modifique nuestro sistema productivo.

Es razonable que ese sea el objetivo que se fije nuestro país, tanto por motivos de compromiso internacional como por el peso que tienen los mercados de los países que están impulsando esa transformación. Si además consideramos que España es uno de los países europeos con mayor dependencia energética, $D$

https://www.ecologique-solidaire.gouv.fr/loi-transition-energetiquecroissance-verte 
lo que se traduce en un gasto medio diario de 69 millones de euros ${ }^{5}$ en importar combustibles fósiles, esta razonabilidad se convierte en necesidad.

Partiendo, pues, de este objetivo, hay que plantearse dos cuestiones. En primer lugar, ¿es realmente necesaria una ley?; en segundo lugar, ¿qué elementos debería incorporar?

\section{1. ¿Por qué una ley de cambio climático?}

$\mathrm{Si}$, como propugno, compartimos la ambición en el objetivo con los países de nuestro entorno, automáticamente llegaremos a la conclusión de que solo una norma con rango de ley puede impulsar este proceso. Y ello es así por motivos económico/empresariales y, también, por motivos sociales y políticos.

Económicamente, la transición energética es un reto similar a otros que ya vivió nuestra economía. Podemos citar los dos más cercanos: en los primeros años de los ochenta, la necesidad de integrarnos en el Mercado Común; en los noventa, el esfuerzo para integrarnos en la Unión Económica y Monetaria y en el área euro. Al igual que en aquellos momentos, la economía y las empresas españolas deberán adoptar una nueva forma de hacer negocios; surgirán problemas de adaptación, pero, también, se abrirán numerosas oportunidades y mercados.

En el caso de la transición energética, el borrador del Plan Nacional Integrado de Energía y Clima (PNIEC) cifra en más de 230.000 millones de euros la inversión necesaria para

5 Saldo neto diario de la balanza energética de España en 2018 según datos de MINECO: https://www.comercio.gob.es/es-ES/comercioexterior/estadisticas-informes/Paginas/Informes-de-Comercio-Exterior. aspx alcanzar sus objetivos en 2030. Un informe de Deloitte $^{6}$ estimaba la inversión 2017-2050 entre 400.000 millones y 500.000 millones de euros, según los escenarios.

Esta inversión dependerá, en gran medida, de la iniciativa privada (según el PNIEC, en más de un $80 \%$ ), y es bueno que así sea si se considera que en el proceso surgirán no pocos riesgos tecnológicos, comerciales y de mercado. Ejemplos de estas oportunidades son los nuevos servicios de eficiencia energética, la movilidad sostenible, el almacenamiento energético, la digitalización eléctrica, nuevas tecnologías limpias en los usos industriales, etcétera.Además, la disponibilidad actual de capitales elimina problemas de falta de financiación.

Es bueno, pues, que en este proceso los mayores riesgos los asuman las empresas. Pero también es necesario recordar que ante inversiones arriesgadas y, en ocasiones, con muy alto componente de inversión inicial y retorno a largo plazo (como, por ejemplo, las infraestructuras energéticas), es fundamental que los riesgos políticos y regulatorios se minimicen. Sencillamente, la política de transición energética no puede gestionarse, a nivel nacional, dependiendo del ciclo político: si así fuera, no cabe duda de que la inversión se realizaría..., pero fuera de España.

Del mismo modo hay que exigir a los poderes públicos que el conjunto del marco regulatorio sea no solo fiable, sino también coherente. Si, como se pide al sector empresarial, hay que avanzar en la descarbonización de todo el sistema, es indudable que las políticas del sector público han de estar alineadas en la misma dirección. Por citar solo algunos ejemplos, no tiene sentido embarcar a las empresas en $\triangleright$

6 Una transición inteligente hacia un modelo energético sostenible para España en 2050: la eficiencia energética y la electrificación (Monitor Deloitte, 2018). 
desarrollar nuevos servicios si la fiscalidad es contraria a ese cambio o la política educativa no fomenta los perfiles y formaciones que se necesitan. Así mismo, tampoco tendría sentido una Administración pública que no tenga sus propios objetivos de descarbonización, o normativas técnicas que no fomenten el nuevo escenario, o, por terminar, velocidades y enfoques diferentes entre las distintas Administraciones territoriales.

La forma práctica de superar parte (nunca todas) de estas limitaciones es, al menos, contar con una ley que gobierne el proceso y dé unas mínimas garantías. Una ley, además, que cuente con un consenso político amplio, y que sobreviva al ciclo político.

Desde el punto de vista social y político, la necesidad de una norma legal es también evidente. Las transiciones económicas tienen costes de ajuste, que derivan de la necesidad de adaptación de partes de la sociedad (regiones, sectores económicos, perfiles profesionales, etcétera). Estos ajustes generan, como es lógico, oposición y pueden hacer fracasar todo el proceso si este no cuenta con tres elementos imprescindibles: una gestión adecuada del tiempo para facilitar la adaptación, un análisis transparente de los costes y beneficios... y, por supuesto, una lealtad y compromiso con los consensos alcanzados.

De nuevo, estas necesidades confluyen en una herramienta que, si no es suficiente, sí que es necesaria: una ley que gobierne el proceso. Una ley permite crear el ritmo necesario y acordar la adaptación y las ayudas a los sectores realmente afectados; en paralelo, define el consenso hacia el que todos los agentes políticos deben lealtad. Por su parte, el análisis de los costes y beneficios garantiza la transparencia y evita que la sociedad asuma pasivos que no responden en realidad al proceso de transición, aunque se pretendan hacer pasar como tales.

\subsection{Elementos para una ley de cambio climático en España}

No existe un formato de ley universal. Incluso dentro de la UE, las diferencias institucionales, sociales y económicas justifican que los contenidos deseables de una ley sean distintos entre países. En general, hay dos posibles modelos: por un lado, leyes de carácter detallado con abundancia de medidas concretas y, por otro lado, leyes paraguas, que buscan más inspirar al resto de normas posteriores que avanzar en los detalles?.

En general, en el caso español, las empresas defensoras de la necesidad de una ley apoyan la primera opción ${ }^{8}$, sin duda conscientes del retraso que experimenta este proyecto en nuestro país y, también, de la necesidad de evitar diferentes enfoques entre las Administraciones que solo llevarían a generar confusión en el proceso.

Quizá para evitar apriorismos sea más constructivo definir los elementos mínimos que, en nuestra opinión, debería tener una ley en nuestro país. A partir de ahí derivaremos propuestas sobre su contenido mínimo.

Un primer elemento es que la ley debe ser clara y finalista, por ello, ha de incorporar objetivos de reducción de emisiones, penetración de renovables y eficiencia cuantificables, a 2030 y 2050 . También incorporar criterios $\triangle$

\footnotetext{
7 Los interesados pueden acudir al reciente estudio de Alina Averchenkova para el Real Instituto Elcano, Legislating for a low carbon and climate resilient transition: learning from international experiences.

8 Ver, por ejemplo, el posicionamiento del Grupo Español de Crecimiento Verde: https://grupocrecimientoverde.org/el-grupo-espanolde-crecimiento-verde-considera-que-el-proyecto-de-ley-de-cambioclimatico-y-transicion-energetica-es-una-buena-propuesta-paraaprovechar-las-oportunidades-economicas-de-avanzar-hacial
} 
rigurosos de revisión de tales objetivos, siempre al alza ${ }^{9}$.

Un segundo elemento es que sea transversal. Para ello, ha de afectar a todos los sectores económicos, no solo al energético, y repartir óptimamente las obligaciones.

Un tercer elemento es que sea una ley marco, pero incluyendo medidas concretas que son imprescindibles. En concreto, hay tres elementos que no deberían faltar:

- Un tratamiento de la fiscalidad ambiental que fije precios relativos compatibles con la transición.

- Una modificación integral (y progresiva) del papel de las Administraciones públicas como compradoras de obras, bienes y servicios, incentivando la compra pública ecológica y liderando con el ejemplo.

- Medidas para incorporar la sostenibilidad a la normativa financiera, siguiendo el ejemplo tanto de la ley francesa como del reciente Plan de Acción de la Comisión Europea sobre finanzas sostenibles y, en concreto, su propuesta de reglamento para una nueva taxonomía financiera.

Un cuarto requisito es que la ley sea creíble y de toda la sociedad. Para ello, en primer lugar, debe partir de un análisis y una metodología públicos y, además, disponer de un comité científico respetado, que dé seguridad a legisladores y sociedad, al tiempo que evite debates faltos de soporte científico. En segundo lugar, se ha de rendir cuentas anualmente de su cumplimiento y avance de modo público, y crear un órgano de control externo con capacidad ejecutiva e independencia de actuación.

9 En estricta coherencia con la necesidad de alcanzar los objetivos firmados por España en el Acuerdo de París.
En paralelo, el Parlamento debe poder analizar la evolución de las medidas.

El quinto requisito es que la ley ha de ser integradora. En una nación con tan alta descentralización administrativa como España es fundamental que el conjunto de Administraciones territoriales avance de una manera relativamente coordinada, que no idéntica. En mi opinión, esto exige que la ley incorpore dos elementos muy importantes.

En primer lugar, un mecanismo de coordinación administrativa en el que se pueda aspirar, al menos, al intercambio de información y la alineación de objetivos. En segundo lugar, una actuación decidida en favor de la coherencia, para lo que hay que introducir instrumentos legales que incentiven económicamente el alineamiento de objetos y políticas, dificultando comportamientos individuales que imponen sobrecostes al proceso e impiden las economías de escala. Un enfoque económico al estilo del que practica la Comisión Europea, que no es otro que la condicionalidad financiera en sus relaciones con los Estados miembros.

El sexto y último requisito es que la ley debe ser justa. Para ello, ha de impulsar prioritariamente las actividades más eficientes económicamente y, en paralelo, facilitar el ajuste de los sectores que se enfrentan a cambios. Pero esa justicia ha de ser bidireccional, esto es, tanta justicia amerita al que reclama ayuda para adaptarse como aquel que exige asumir solo los costes estrictamente necesarios del proceso. Es por ello que este criterio de transición se debe objetivar, basar en la evidencia y los datos transparentes: solo así se ayudará al que lo necesita y, al tiempo, se evitará que se perpetúen costes o subvenciones injustificadas.

De nuevo, en este punto, la condicionalidad parece un requisito irrenunciable. La ayuda $\triangleright$ 
a la transición justa no puede ser una compensación para seguir haciendo lo mismo, sino, como el propio concepto indica, para transitar a un nuevo modelo. En este sentido, la ley ha de prever tal carácter condicional de las eventuales medidas de apoyo.

En definitiva, los elementos mencionados deberían formar parte de una ley de cambio climático eficaz y perdurable para nuestro país. Obvia decir que no son, ni mucho menos, los únicos, pero quizá sí los más importantes.

\section{Algunas claves para el debate que nos espera}

No cabe duda de que el debate y aprobación de una ley de transición energética y cambio climático en nuestro país es uno de los primeros proyectos que cabe esperar de la legislatura que surge de las elecciones de noviembre de 2019. Y esto es así no solo por la voluntad del partido socialista, cuyo Gobierno ya presentó un primer anteproyecto, sino porque la mayoría del resto de partidos representativos apoya esta idea.

El hecho de que a lo largo de la pasada década estos partidos políticos hayan realizado su propia «transición energética» y ahora coincidan en la necesidad y urgencia del proceso no significa (y menos aún en un país como España) que el proyecto no vaya a generar un intenso debate. En este último apartado del artículo repasaré algunos de los debates que más recurrentemente surgen al tratar el proceso de transición, y que sin duda se repetirán en el futuro cercano.

Aunque me centraré en los aspectos vinculados con un enfoque más técnico-económico, no quiero dejar de hacer una reflexión inicial sobre el principal riesgo que, a mi juicio, se plantea. Me refiero en concreto al lícito, pero arriesgado, esfuerzo de convertir la política de transición energética en una herramienta para cuestionar el modelo de economía de mercado. Es lícito, como todo posicionamiento político, pero supone convertir la política climática en una herramienta que trasciende su objetivo $y$, por tanto, arriesga generar rechazos y convertirla en un campo de batalla ideológico.

La realidad ambiental y sus urgencias justifican que, aunque solo sea por pragmatismo, libremos a la transición energética de convertirse en otra batalla de esta ya secular guerra. Se haría un flaco favor a la lucha contra el cambio climático si se desaprovechara la ocasión para convertirla en un esfuerzo conjunto y no partidista. Eso no significa que en su seno no convivan instrumentos o políticas que excedan su impacto puramente energético (un ejemplo es el énfasis que algunos asignan al autoconsumo como herramienta para la descentralización de la propiedad energética), pero quizá lo más práctico sea juzgar estas medidas solo en lo que demuestren que sirven para luchar contra el cambio climático.

\subsection{El esfuerzo proporcionado}

Uno de los argumentos que habitualmente se utilizan para moderar o limitar la necesidad de la transición energética en España es el del «esfuerzo proporcionado». En términos generales, este argumento se utiliza explicando la irrelevancia de las emisiones de GEI generadas en España (0,23 GT de $\mathrm{CO}_{2}$ en 2017 vs. 36,2 GT de $\mathrm{CO}_{2}$ estimadas para el mundo) ${ }^{10}$, y se completa con la reflexión sobre el escaso peso de las emisiones de la Unión Europea versus $\triangleright$

10 Global Carbon Project 2018. 


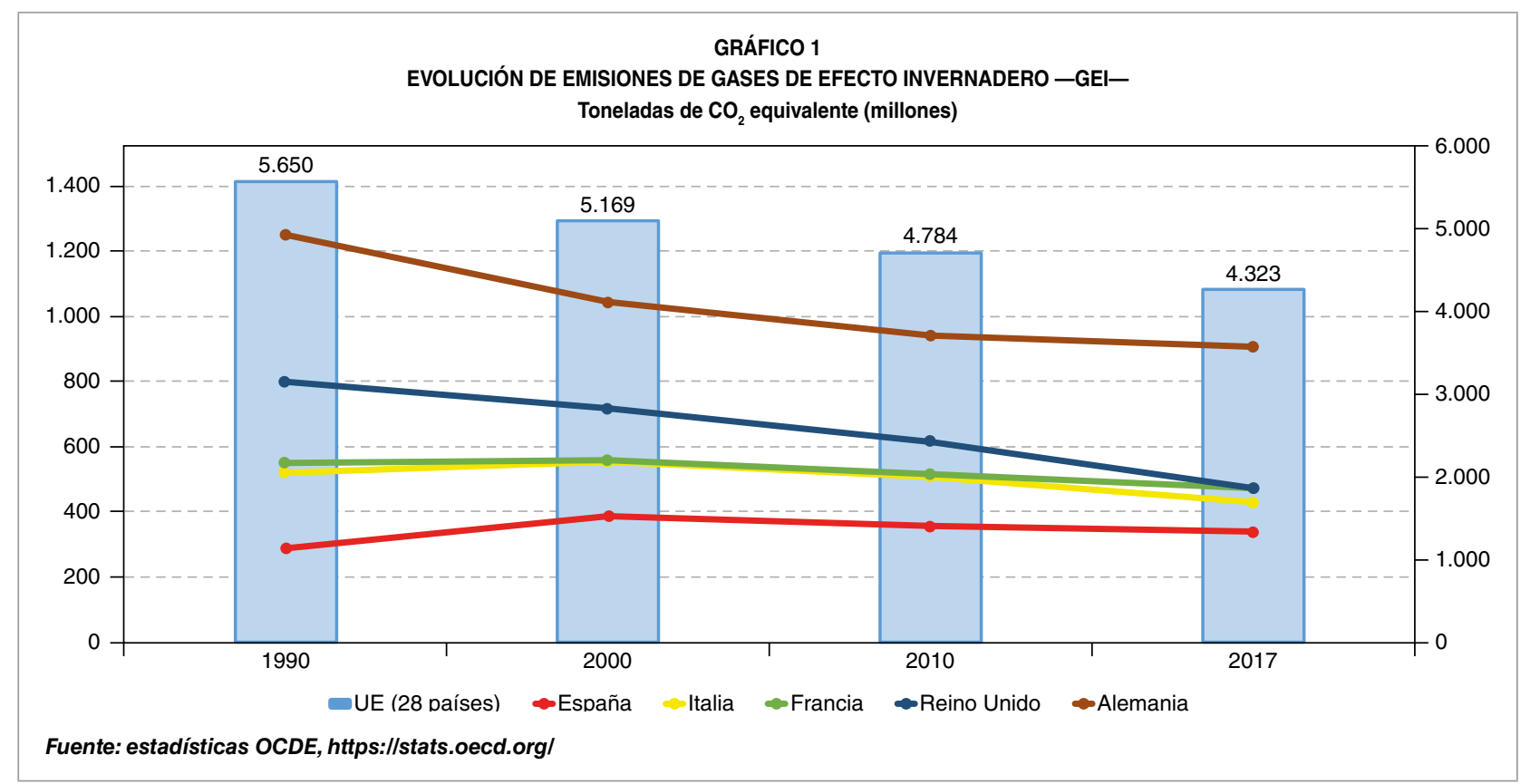

el total mundial (apenas el $10 \%$, y decreciendo). Aunque no se suele concluir el argumento (al menos públicamente), la cadena de razonamiento lleva a una conclusión clara: tendría más sentido no hacer nada y esperar a que se muevan los grandes bloques. En su vertiente más edulcorada, el corolario es más moderado: reduzcamos nuestro nivel de ambición (o, siguiendo la misma lógica: seamos aún más irrelevantes).

Frente a esta posición, equivalente a asumir una postura de «polizón» o «free rider» propia de los bienes públicos, cabe recordar que incluso al margen de criterios de responsabilidad ética, desde un punto de vista económico, solo se justificaría si los costes de actuar superan a los beneficios. En definitiva, si se considera que este cambio estructural de la economía aporta pérdidas netas.

No es el objetivo de este artículo entrar en detallar la oportunidad económica de la transición, avalada por múltiples estudios. Es más, en buena parte de ellos se identifica a España como uno de los países que más pueden ganar de la misma ${ }^{11}$, basta recordar nuestra total carencia de recursos energéticos convencionales o la aportación negativa secular de nuestra balanza energética.

En todo caso, tampoco conviene aceptar la tesis del «desproporcionado» esfuerzo sin una mínima comprobación. Para ello, basta reflexionar sobre un dato: mientras que entre $1990 \mathrm{y}$ 2017 las emisiones de GEI en la UE-28 se redujeron un $23,5 \%^{12}$ (incluyendo las reducciones del 40,5\% de Reino Unido, 14,5\% en Francia o $27,5 \%$ de Alemania), las de España... aumentaron en un $18 \%$. De hecho, España es uno de los seis países europeos que han aumentado sus emisiones desde 1990 (junto a Portugal, Austria, Chipre, Irlanda y Malta) ${ }^{13}$.

Los datos anteriores muestran que, quizá, el esfuerzo no sea tan desproporcionado como a veces se escucha.

11 Eurofound (2019)

12 Greenhouse gas emissions, OECD.Stat, https://stats.oecd.org/ Index.aspx?DataSetCode=AIR GHG\#

13 European Environmental Agency (2019). 


\subsection{Competitividad y descarbonización}

Un segundo argumento que se suele mencionar es el de la competitividad. De hecho, en ocasiones se intenta contraponer este concepto al de transición, como si fueran realidades incompatibles.

De nuevo aquí hay múltiples consideraciones a realizar sobre esta simplificación del proceso, empezando por el auténtico sinsentido que supondría que la Unión Europea en su conjunto o países con vocación de liderazgo económico mundial, como Francia, Reino Unido o Alemania, practicaran esta especie de suicidio económico por contagio. También se podría hacer una reflexión sobre el propio concepto de competitividad, que da lugar a numerosos estudios internacionales de grandes variables ${ }^{14}$ pero que, por desgracia, en numerosas ocasiones queda reducido en los debates internos al impacto a corto plazo en una variable concreta en un sector económico no menos concreto..., confundiendo así la parte con el todo.

La realidad es que, al menos en España, este debate se ha limitado al de la competitividad de la factura eléctrica (ni siquiera energética) para un segmento muy concreto de empresas, identificadas como electrointensivas ${ }^{15}$. Este enfoque es muy parcial, aunque es razonable analizarlo, pues entronca con las preocupaciones europeas de evitar que la industria se vea penalizada frente a otras zonas del mundo, provocando una deslocalización industrial (la llamada «fuga de carbono»).

\footnotetext{
14 Sin ir más lejos, cabe recordar el Global Competitiveness Index del Foro Económico Mundial.

15 Una necesaria lectura para entender la realidad de los costes eléctricos y el peso de sus componentes en los distintos sectores industriales son los estudios de la Comisión Europea alentados por el comisario Arias Cañete precisamente para aportar datos contrastables y huir de los prejuicios. El más reciente es la Comunicación COM (2019) 1 final sobre «Energy prices and costs in Europe», de enero de 2019.
}

De nuevo, no es este el lugar para analizar en detalle este asunto ${ }^{16}$, pero sí para animar al lector a realizar su propio análisis y exigir transparencia en el debate. A menudo se oculta que Europa (y España) tiene ya un amplio conjunto de medidas diseñadas para evitar este riesgo, desde la asignación gratuita de derechos de emisión a las industrias más emisoras (que se pueden vender a otras empresas) hasta la concesión de ayudas para compensar posibles subidas en el precio de su electricidad.

En el caso de España, estas medidas incluyeron, hasta hace poco, un importante apoyo económico en forma de pagos por interrumpibilidad, que en los peores momentos del déficit tarifario eléctrico (2013) fueron aumentados a 677 millones de euros (cargados contra la factura eléctrica del resto de usuarios). La intervención de la Unión Europea ha obligado a la reducción de este mecanismo y orientado el mismo hacia ayudas específicas, transparentes y evaluadas públicamente, al parecer dentro de un bienvenido estatuto de empresa electrointensiva que se pretende aprobar en breve.

Cabe también mencionar la nueva vía impulsada por la presidenta de la Comisión Europea, Von der Leyen. Ha anunciado el objetivo de crear un nuevo impuesto al $\mathrm{CO}_{2}$ en frontera (border carbon tax), precisamente para evitar la entrada en el mercado europeo de productos artificialmente competitivos al no cumplir con los compromisos de descarbonización.

Retomando los criterios identificados al inicio de este artículo, hay que reivindicar en este punto tanto la necesidad de actuar como la necesidad de transparencia. En un entorno de transición $\triangleright$

\footnotetext{
16 Un enfoque interesante por su seriedad es el estudio elaborado en 2016 por el Instituto Vasco de Competitividad de la Fundación Deusto "Precios de la energía y competitividad industrial», que recuerda que hay muchos más componentes que el coste de la energía al considerar la competitividad de un sector (tamaño de las empresas, intensidad inversora, productividad, mercados, capacidad tecnológica, grado de competencia, coste de mano de obra, canales de comercialización...).
} 
energética se debe evitar perjudicar a empresas industriales de modo innecesario o inasumible y serán necesarios entornos de apoyo público y colaboración con la industria para facilitar una transición ordenada. En paralelo, la mejor manera de implantar estas medidas de ayuda es mediante una evaluación previa objetiva de la necesidad de apoyo y, solo en caso de revelarse necesarias, el diseño de medidas que garanticen que ese apoyo es compatible con conseguir un proceso de transición en esos sectores.

\subsection{El argumento de la neutralidad tecnológica}

El tercer y último argumento que quiero destacar es el relativo a la necesidad de respetar la «neutralidad tecnológica», concepto que sin duda se escuchará en los debates sobre la ley.

La neutralidad tecnológica es, sin duda, un enfoque que debe propugnarse al discutir la futura ley y los planes de transición. De nuevo, hay que acordar primero qué es lo que se entiende por ese término: así, debemos evitar una aproximación basada en un enfoque político (como la que presenta la neutralidad tecnológica como alternativa a un supuesto «intervencionismo tecnológico») y buscar un concepto técnico y medible. Bajo este último prisma, la neutralidad tecnológica exige ser neutral entre tecnologías y combustibles que, a corto y medio plazo, garanticen que se cumplen los objetivos de descarbonización y reducción de emisiones.

Hay que tener en cuenta que todo el esfuerzo de la transición debe servir para alcanzar unos objetivos de reducción de emisiones a 2030 y, sin duda, de emisiones netas cero a 2050. En ese escenario pueden y deben subsistir tecnologías de transición, siempre que ayuden a cumplir los objetivos intermedios finales.
Sin embargo, lo que no tiene sentido es confundir esas tecnologías con opciones definitivas a 2050.

La neutralidad tecnológica debe, pues, aplicarse sobre las tecnologías eficaces disponibles en cada momento, y evitar en paralelo incurrir en inversiones erróneas de largo plazo con un efecto lock-in a futuro en tecnologías que solo son de transición.

Para evitar debates estériles sería útil contar con el comité de expertos científicos antes mencionado como uno de los elementos deseables de la ley. También lo sería centrar los apoyos iniciales a tecnologías inmaduras en programas de $\mathrm{I}+\mathrm{D}+\mathrm{i}$ y pasar los apoyos al entorno de la política energética solo cuando se tenga certeza de la viabilidad a medio plazo de la solución tecnológica.

En definitiva, sí a la neutralidad tecnológica, basada en criterios objetivos y técnicos, nunca políticos ni ideológicos.

\section{Conclusiones}

El diseño y ejecución de un modelo de transición energética para la economía española está motivado no solo por los compromisos internacionales asumidos por nuestro país, sino, en paralelo, por suponer un programa de cambio estructural que puede aportar grandes beneficios en términos de inversión y creación de empleo.

Desde este enfoque, la transición energética es un reto de toda la sociedad, al nivel de los que ya fuimos capaces de superar con éxito, como la entrada en el Mercado Común o la integración en el euro.

Al igual que aquellos retos, el cambio será tanto más eficiente y exitoso cuanto más unida esté la sociedad y sus representantes $\triangleright$ 
políticos en torno a esta idea. Surge así la necesidad de una figura de rango legal, una ley de transición energética que, al estilo de las que ya existen en países de nuestro entorno, o de la que se va a dotar la Unión Europea, fije de modo consensuado los objetivos, los instrumentos, los tiempos y, también, las medidas para ayudar a los que lo necesiten.

La transición energética es, pues, mucho más que un ejercicio obligado de solidaridad intergeneracional o de puro compromiso ético con el medio ambiente y el planeta en que vivimos, y que nos sustenta desde hace millones de años. Es una oportunidad económica, muy favorable para un país como España con una gran dotación de recursos energéticos no fósiles (viento, sol y agua), capital humano y un gran posicionamiento y know-how en sectores de futuro (como la industria renovable).

Una ley que impulse y gobierne ese proceso, que aporte transparencia, información, estabilidad regulatoria y solidaridad, sería, sin duda, la mejor manera de enfocar este reto y maximizar sus beneficios. Esperemos que el futuro debate político esté al nivel del reto y, también, del compromiso actual de gran parte de los ciudadanos.

\section{Bibliografía}

Averchenkova, A. (2019). Legislating for a low carbon and climate resilient transition: learning from international experiences. Real Instituto Elcano (colección Elcano Policy Paper 3/2019).

Averchenkopva, A., Fankhauser, S., y Nachmany, M. (eds.) (2017). Trends in Climate Change legisltation. Edward Elgar.

Climate Change Act 2008. Legislation Government UK. http://www.legislation.gov.uk/ukpga/2008/27/ contents
Comisión Europea (2019). Comunicación COM (2019) 1 final sobre Precios y Costes de la Energía en Europa. https://ec.europa.eu/transparency/regdoc/rep/1/2019/EN/COM-2019-1-F1-ENMAIN-PART-1.PDF

Comisión Europea (2019). Comunicación COM (2019) 640 final sobre el Pacto Verde Europeo. https://ec.europa.eu/transparency/regdoc/ rep/1/2019/ES/COM-2019-640-F1-ES-MAINPART-1.PDF

Díaz, A. C., Larrea, M., Kamp, B., y Álvarez, E. (2016). Precios de la Energía y Competitividad Industrial. Instituto Vasco de Competitividad-Fundación Deusto.

Eurofound (2019). Energy scenario: Employment implications of the Paris Climate Agreement. Publications Office of the European Union, Luxemburgo.

European Environmental Agency (2019). Annual European Union greenhouse gas inventory 1990-2017 and inventory report 2019. Bruselas, Bélgica.

Lázaro, L., González, C., y Escribano, G. (2019). Los españoles ante el cambio climático. Apoyo ciudadano a los elementos, instrumentos y procesos de una Ley de Cambio Climático y Transición Energética. Real Instituto Elcano.

LOI n. ${ }^{\circ}$ 2015-992 du 17 août 2015 relative à la transition énergétique pour la croissance verte (1), NOR: DEVX1413992L. https://www.legifrance. gouv. fr/affichTexte.do? cidTexte=JORFTEXT 000031044385

Monitor Deloitte (2018). Una transición inteligente hacia un modelo energético sostenible para España en 2050: la eficiencia energética y la electrificación. https://perspectivas.deloitte.com/modelo-energetico-sostenible?utm_campaign=Descarbonizacion-2018\&utm_source=CTA-DTT.com

World Economic Forum (2019). The Global Competitiveness Report 2019. http://www3.weforum. org/docs/WEF_TheGlobalCompetitivenessReport2019.pdf 